\title{
¿Peleamos o negociamos? La argumentación de los padres y las madres con sus hijos e hijas adolescentes durante los conflictos familiares
}

\author{
Nieves Correa Rodríguez, Juan Rodríguez, Esperanza Ceballos y Miriam Álvarez \\ Universidad de La Laguna (España)
}

La polarización de posturas en los conflictos entre padres e hijos/as adolescentes supone una amenaza para la convivencia familiar. Durante los procesos de negociación es necesario desplegar una adecuada argumentación poniendo en marcha el perspectivismo como recurso imprescindible para facilitar el logro de acuerdos satisfactorios para todos. En este estudio se investiga mediante metodología observacional la capacidad de adoptar otras perspectivas durante un conflicto. Para ello 29 familias (constituidas por el padre, la madre y un/a hijo/a adolescente) fueron grabadas mientras discutían acerca de un conflicto real. Se analizaron la frecuencia y la duración de cinco niveles perspectivistas (Yo simple; Yo Razonado; el Otro como Obstáculo; el Otro Añadido; y Nosotros) en el discurso familiar, en función de cada uno de los miembros de la tríada, así como las relaciones que se establecen entre sí. Los resultados muestran un moderado perspectivismo en las discusiones familiares, predominando los niveles «Yo Razonado» con explicaciones sobre la propia posición, y «el Otro como Obstáculo» con razones para rebatir las posiciones contrarias. Los padres, en general, conceden mayor oportunidad al perspectivismo que los adolescentes, especialmente las madres, quienes muestran más sensibilidad con los hijos/as al emplear niveles perspectivistas y de argumentación más elevados.

Palabras clave: Conflictos familiares, padres-adolescentes, argumentación oral, perspectivismo, negociación.

Shall we quarrel or deal? The argumentation processes of parents and adolescents during family conflicts. The polarization of postures in conflicts among parents and adolescents involve a threat for family harmony. Skills for argumentation and perspective taking are required to enable the achievement of satisfactory agreements during the processes of negotiation. The purpose of this paper has been to analyze the skills of parents and adolescents to adopt other perspectives when discussing a conflict by means of observational methodology. The study was carried out with 29 families (constituted by father, mother and an adolescent son or daughter) who were recorded while they argued about a real conflict. Frequency and duration of five levels of perspective-taking were registered (Myself; Reasoned myself; the other like an obstacle; the other adding to me; and us) in the discourse of the family, and also in the different members of the triad, considering their interrelations. The results were showing a moderated level of perspective-taking in family discussions, predominating the levels Reasoned myself, focused on explaining own perspective, and The other like an obstacle, focused on reasons to contest the perspective of the other. In general parents showed more perspective-taking. Particularly the mothers exhibited more sensitivity with her adolescent son or daughter, providing higher levels of perspective-taking and argumentation.

Key words: Family conflicts, parents-adolescents, oral argumentation, perspective-taking, negotiation.

Correspondencia: Dra. Nieves Correa Rodríguez. Departamento de Psicología Evolutiva y de la Educación. Facultad de Psicología. Universidad de La Laguna. Campus de Guajara, s/n. C.P. 38071. La Laguna (España). E-mail: $\underline{\text { mcorrea@ull.es }}$ 
Los conflictos entre padres e hijos en la adolescencia, aunque valorados en general como negativos, resultan episodios fundamentales para el desarrollo de los hijos. La confrontación entre padres e hijos adolescentes se debe básicamente a que los hijos quieren renegociar la relación con sus padres hacia una mayor horizontalidad (García y Peralbo, 2001; Parra y Oliva, 2002), evidenciándose la necesidad de una nueva forma de gestionar los conflictos en las familias; desde una relación directiva y unilateral, en la que los padres dominan el discurso con preguntas directas, propuestas y órdenes para sus hijos, hacia una relación más simétrica en la que los hijos aumentan su participación con propuestas no prescriptivas, justificaciones y contraargumentos (Hofer, Sassenberg y Pikowsky, 1999).

Como en otras interacciones cotidianas, con frecuencia lo que se pretende es persuadir al otro de que la postura propia es la mejor, por lo que los interlocutores suelen estar más preocupados por lograr que su propuesta resulte vencedora que por determinar lo que es más justo o razonable. La persuasión está presente en los hijos ya desde muy temprana edad y en principio se encuentra plasmada en las justificaciones para apoyar la postura propia. Sin embargo, la aparición de los contraargumentos (razones para rechazar la postura ajena) es un aspecto clave para revelar el desarrollo de las capacidades argumentales complejas y el aumento de la simetría en las relaciones parento-filiales, encontrando que en la adolescencia el uso de contraargumentos llega incluso a ser mayor que el de los padres (Correa et al., 2009).

Este aumento de la oposición adolescente, aunque normativo y necesario, puede provocar serias barreras para la resolución de los conflictos cuando padres e hijos se atascan en sendos posicionamientos, haciendo inviables la negociación y el logro de compromisos (Leitão, 2003). En cambio, la solución negociada de los conflictos requiere capacidades argumentales complejas tanto en los padres como en los hijos: se trata de que ambas partes expongan sus puntos de vista respectivos mientras paralelamente intentan entender la propuesta del otro e incorporarla a la propia (Correa, Ceballos, Correa y Batista, 2003). Mostrando así capacidades perspectivistas de alto nivel que implican ponerse en la perspectiva mental ajena, contrastarla con la propia, y complementar ambos puntos de vista para la búsqueda de una solución nueva y negociada.

Parece razonable esperar que la argumentación y el perspectivismo se fomenten más en contextos familiares participativos en donde se dediquen esfuerzos a la construcción de una salida conjunta, favoreciendo un mayor conocimiento y comprensión mutuos y, muy posiblemente, potenciando el deseable desarrollo cognitivo y socioemocional en la adolescencia. En el lado opuesto se situarían los contextos familiares competitivos y de emotividad negativa donde se trata de imponer la propia visión a los otros, denotando un escaso nivel perspectivista. En estos últimos las partes en liza quedarían ancladas en la fase de posicionamiento durante el proceso de 
negociación (Medina, Dorado, de Cisneros, Arévalo y Munduate, 2004), dando lugar a patrones seriales de quejas y ataques (Johnson y Roloff, 2000) en los que la tensión emocional negativa aparece con fuerza (Gottman y Levenson, 2000).

Es por ello que consideramos fundamental desvelar la aparición de discursos argumentativos que evidencien perspectivismo, tanto en los padres como en los hijos adolescentes. Pues mientras ambos permanezcan inmovilizados en sus respectivas posturas, el conflicto se aboca a un ciclo infinito de desgaste y distancia emocional. Para identificar el perspectivismo en el discurso de padres e hijos, partimos de que la argumentación menos perspectivista consiste en la simple defensa de la propuesta propia o en la negación de la propuesta del otro, mientras que la argumentación más perspectivista se orienta a enlazar la propuesta propia con la del otro para construir una propuesta nueva que aúne lo mejor de cada uno y satisfaga las necesidades de todos. En esta línea el propósito de este estudio es analizar las categorías argumentales propias de diferentes niveles perspectivistas en el discurso de las madres, los padres y los hijos e hijas adolescentes acerca de conflictos familiares reales, así como las relaciones entre dichos niveles perspectivistas y los diferentes miembros de la tríada.

\section{MÉTODO}

\section{Participantes}

La muestra está compuesta por veintinueve tríadas (padre-madre-hijo/a adolescente) que fueron grabadas en vídeo. La edad de los progenitores oscilaba entre los 40 y los 55 años. Los hijos e hijas se encontraban en la adolescencia inicial e intermedia $(M=14.4)$ con edades entre los trece $(27.6 \%)$, los catorce $(20.7 \%)$, los quince (31\%) y los dieciséis años $(20.7 \%)$.

\section{Procedimiento}

El contacto con las familias participantes se hizo a través de estudiantes de la Facultad de Educación de la Universidad de La Laguna que se prestaron a colaborar voluntariamente. Los estudiantes se desplazaron a los hogares e informaron a las familias sobre cómo realizar las grabaciones. La tríada familiar elegía un conflicto familiar que los tres coincidieran en calificar como frecuente e intenso emocionalmente, y que además hubiese ocurrido en las dos semanas anteriores a la grabación. Tras un breve ensayo de muestra, la familia se quedaba a solas, siendo alguien cercano a la familia el encargado de la grabación. Aunque no se estableció un límite de tiempo, las grabaciones duraron entre 12 y 15 minutos.

\section{Unidades de medida}

Se elaboró un sistema de categorías de discurso argumental partiendo de estudios previos que analizan la argumentación oral en la gestión de conflictos entre 
padres e hijos (Correa et al., 2009; Hofer, Youniss y Noack, 1998). Finalmente se conformaron un total de 16 categorías de discurso argumental organizadas en cinco niveles de perspectivismo: "Yo simple”, "Yo razonado”, “El otro como obstáculo”, "El otro añadido" y el "Nosotros" (Tabla 1).

Tabla 1. Niveles perspectivistas, categorías argumentales y ejemplos

\begin{tabular}{|c|c|c|}
\hline $\begin{array}{c}\text { Niveles } \\
\text { Perspectivistas }\end{array}$ & Categorías Argumentales & Ejemplos de las categorías \\
\hline \multirow[b]{2}{*}{ "Yo Simple" } & $\begin{array}{l}\text { Oposición Simple: Rechazar un } \\
\text { argumento sin justificarlo. }\end{array}$ & $\begin{array}{l}\text { "No, nunca he hecho eso"; "Eso no es verdad"; } \\
\text { "Paso de tus charlas". }\end{array}$ \\
\hline & $\begin{array}{l}\text { Propuesta Prescriptiva: Solicitar, o } \\
\text { exigir algo al otro. }\end{array}$ & $\begin{array}{l}\text { "Tienes que estudiar más y ver menos la tele"; } \\
\text { "Mañana pones el ordenador en el salón, no quiero } \\
\text { que esté en tu cuarto". }\end{array}$ \\
\hline \multirow[t]{2}{*}{ "Yo Razonado" } & $\begin{array}{l}\text { Propuesta no prescriptiva: Expresar las } \\
\text { propuestas como opiniones personales. }\end{array}$ & $\begin{array}{l}\text { "Yo creo que hay que discutir lo del dinero"; "Pienso } \\
\text { que hay que hablar mejor lo de salir hasta tan tarde"; } \\
\text { "Creo que tengo edad para poder salir hasta más } \\
\text { tarde". }\end{array}$ \\
\hline & $\begin{array}{l}\text { Justificación: Apoyar la propuesta con } \\
\text { razones. }\end{array}$ & $\begin{array}{l}\text { "Hay que pensar lo de ir a la fiesta porque tenemos } \\
\text { muchos gastos"; "Porque mis amigas también salen"; } \\
\text { "Porque es bueno que hagamos cosas juntos". }\end{array}$ \\
\hline \multirow{3}{*}{$\begin{array}{l}\text { "El Otro como } \\
\text { Obstáculo" }\end{array}$} & $\begin{array}{l}\text { Contraargumento: Aportar razones en } \\
\text { contra de la postura del otro. }\end{array}$ & $\begin{array}{l}\text { "Padre: En mis tiempos no se podía llegar tarde. Hijo: } \\
\text { Pues los tiempos cambian"; "Padre: Eres aún muy } \\
\text { pequeña para salir. Hija: Mi hermana salía a esta } \\
\text { edad"; "Madre: Creo que estás demasiado tiempo } \\
\text { chateando. Hija: Que esté en el ordenador no quiere } \\
\text { decir que esté chateando." }\end{array}$ \\
\hline & $\begin{array}{l}\text { Preguntas de Control: Realizar preguntas } \\
\text { dirigidas a controlar las actividades de } \\
\text { tipo más comportamental. }\end{array}$ & $\begin{array}{l}\text { ¿Con quién vas a la fiesta?”; “¿Cuánto tiempo } \\
\text { estuviste anoche en el ordenador?; “ ¿A qué horas vas } \\
\text { a volver?". }\end{array}$ \\
\hline & $\begin{array}{l}\text { Respuesta Simple: Responder a una } \\
\text { pregunta escuetamente sin aportar } \\
\text { razones que impliquen aceptar o rechazar } \\
\text { algo. }\end{array}$ & "Voy con Laura."; “Creo que si”; "No, nunca." \\
\hline \multirow{3}{*}{ "El Otro Añadido" } & $\begin{array}{l}\text { Persuasión Simple: Tener en cuenta la } \\
\text { perspectiva del otro. }\end{array}$ & $\begin{array}{l}\text { “Tú no tienes forma de ir a la fiesta con tus amigos? } \\
\text { Pues nosotros te acercamos”; “ ¿No decías que querías } \\
\text { hablar más con tus amigas? Pues puedes". }\end{array}$ \\
\hline & $\begin{array}{l}\text { Persuasión Compleja: Razonar desde la } \\
\text { perspectiva del otro mostrándole las } \\
\text { consecuencias negativas de su postura } \\
\text { para hacer valer la propia. }\end{array}$ & $\begin{array}{l}\text { "Si llegas muy tarde es muy probable que al día } \\
\text { siguiente no puedas jugar el partido de fútbol que tanto } \\
\text { deseabas"; "Si no haces los deberes hoy, mañana } \\
\text { tendrás muchas cosas que hacer". }\end{array}$ \\
\hline & $\begin{array}{l}\text { Pedir Opinión: Solicitar al otro que } \\
\text { exprese su opinión en el tema a discutir. }\end{array}$ & $\begin{array}{l}\text { “¿Y tú qué dices sobre esto?”; “¿Por qué crees que te } \\
\text { mereces una salida a la fiesta?”; “¿Pero, qué crees } \\
\text { que puedo hacer?". }\end{array}$ \\
\hline \multirow{4}{*}{ "Nosotros" } & $\begin{array}{l}\text { Retroalimentación: Verificar que el } \\
\text { mensaje ha llegado adecuadamente. }\end{array}$ & “¿Vale?”; “Lo entiendes?”. \\
\hline & $\begin{array}{l}\text { Hacer Concesiones: Aceptar las } \\
\text { propuestas, iniciativas o argumentos del } \\
\text { otro. }\end{array}$ & $\begin{array}{l}\text { "Si"; "Vale"; "Claro que si'”; "Bueno, está bien, lo } \\
\text { dejamos así"; "Como tú quieras". }\end{array}$ \\
\hline & $\begin{array}{l}\text { Plantear Alternativas: Ofrecer nuevas } \\
\text { opciones para alcanzar una solución al } \\
\text { conflicto. }\end{array}$ & $\begin{array}{l}\text { "Podrías salir hasta las 21:00 y luego venir con } \\
\text { nosotros en el coche", "Una solución sería utilizar } \\
\text { internet en vez del teléfono para bajar la factura"; } \\
\text { "Podríais elegir un día cada una la hora de utilizar el } \\
\text { baño". }\end{array}$ \\
\hline & $\begin{array}{l}\text { Propuesta Conciliadora: Integrar } \\
\text { posturas, modificar la postura inicial } \\
\text { incorporando parte de la perspectiva del } \\
\text { otro para acercar posiciones, alcanzar } \\
\text { acuerdos, solucionar el conflicto. }\end{array}$ & $\begin{array}{l}\text { "Creo que si limpiamos la casa entre todos, } \\
\text { terminaremos antes y podrás salir lo antes posible"; } \\
\text { "Entiendo que quieras jugar con la PlayStation, pero } \\
\text { antes lleguemos a un acuerdo sobre el tiempo de uso"; } \\
\text { "Puedes elegir la ropa, claro, pero cuenta conmigo, te } \\
\text { puedo aconsejar"; "Vale, llegaré cuando acordemos, } \\
\text { pero la hora la elegimos entre los dos". }\end{array}$ \\
\hline
\end{tabular}


El nivel "Yo simple" representa el nivel de perspectivismo más bajo. El discurso gira en torno a la perspectiva propia y se ofrecen al interlocutor posturas cerradas sin aportar explicación alguna. "Yo razonado" continua en torno a la perspectiva propia pero se aportan razones. En ambos niveles, el objetivo es marcar la posición del hablante en el conflicto y hacer valer su postura ante todo. En el nivel "El otro como obstáculo" se ve al otro como un impedimento para que su posición quede legitimada. Es decir, escucha al otro pero con el fin de ofrecerle un argumento para afirmar su propia posición y debilitar la ajena. En "El otro añadido" se percibe al otro como un yo con mente y se realiza el esfuerzo de ponerse en su lugar para saber qué es lo que puede pensar el otro, aunque el objetivo es poder brindarle estratégicamente una respuesta que le sitúe más cerca de la posición propia. El último nivel de perspectivismo "Nosotros" está orientado hacia la búsqueda de soluciones consensuadas y está abierto a otras posturas. Tiene en cuenta la posición del otro para poder negociar, escuchando los intereses del otro y procurando conjugarlos con los propios, renunciando a parte de los suyos. De esta forma puede tender puentes entre las posturas de ambos, evitando la confrontación o buscando formas dialogadas de resolución de las diferencias.

\section{Registro de datos}

Los vídeos fueron codificados con el programa Match Vision Studio 3.0 (Perea, Alday y Castellano, 2006). Se realizó un registro activado por transiciones y los datos se recogieron como eventos con tiempo. Así se pudo obtener una descripción precisa de la frecuencia, la duración y el orden de las conductas (Bakeman y Quera, 1996; Bakeman, Deckner y Quera, 2005).

Se entrenó para la codificación a dos observadoras empleando fragmentos de los vídeos de ensayo. Los coeficientes de la Kappa de Cohen para valorar la fiabilidad interjueces fueron siempre superiores a 0.75 .

\section{RESULTADOS}

Se adoptó un diseño nomotético (Anguera, Blanco y Losada, 2001) en los análisis agrupados realizados para la tríada (padre-madre e hijo/a). Se empleó el programa GSQ de Bakeman y Quera (1996) para obtener la frecuencia de ocurrencia y duración relativa de los niveles perspectivistas de las tríadas (padre-madre e hijo/a). Por tanto, no se trata de unidades de tiempo, sino de porcentajes de ocurrencia y duración.

Se empleó también el programa SPSS-15 para la comparación de las medias de niveles de perspectivismo de los distintos miembros de la tríada familiar mediante la prueba Wilcoxon para muestras relacionadas. Para establecer las correlaciones entre los niveles perspectivistas de los miembros de la tríada se utilizó la rho de Spearman. 
Análisis de la frecuencia y duración de los niveles perspectivistas de los miembros de la tríada

Tal como se aprecia en la tabla 2 de forma global los niveles perspectivistas que presentan un mayor porcentaje de ocurrencia relativa son el "Yo razonado", aportando explicaciones sobre el planteamiento propio, y "El otro como obstáculo", rebatiendo las tesis contrarias. El nivel "Yo razonado" presenta valores significativamente superiores al resto de niveles "Yo simple" $(z=-4.703 ; p<.01)$; "El otro como obstáculo" $(z=-2.498 ; p<.01)$; "El otro añadido" $(z=-4.703 ; p<.01)$; y, "Nosotros" $(z=-4.703 ; p<.01)$. La frecuencia del nivel "El otro como obstáculo", centrado en el ataque a los argumentos contrarios, es significativamente superior a las de los niveles "Yo simple" ( $z=-4.703 ; p<.01)$, "El otro añadido" $(z=-4.509 ; p<.01)$ y "Nosotros" $(z=-4.703 ; p<.01)$. Además, la frecuencia del nivel "Nosotros”, que construye un nuevo planteamiento incorporando las posturas de los otros, es significativamente inferior a las de los niveles "Yo simple" $(z=-4.349 ; p<.01)$, ocupado en la enunciación, sin explicación, de la propia postura y "El otro añadido" $(z=-4.517 ; p<.01)$, que tiene en cuenta las ideas del interlocutor para usarlas estratégicamente en favor propio.

Respecto a la duración relativa, los resultados son similares. Los miembros de la tríada emplean más tiempo en la justificación de sus planteamientos, resultando el nivel "Yo razonado" significativamente superior a los otros niveles perspectivistas: "Yo simple" ( $z=-4.703 ; p<.01) ;$ "El otro como obstáculo" $(z=-3.968 ; p<.01)$; "El otro añadido" ( $z=-4.703 ; p<.01)$; y "Nosotros" $(z=-4.703 ; p<.01)$. El tiempo destinado a atacar la posición del otro, propio del nivel "El otro como obstáculo", ofrece diferencias significativas con el resto de los niveles "Yo simple" $(z=-4.314 ; p<.000)$ "Nosotros", $(z=-4.703 ; p<.01) ;$ y “El otro añadido" $(z=-4.444 ; p<.01)$. El nivel "Nosotros", caracterizado por la búsqueda de soluciones compartidas, presenta valores significativamente inferiores con respecto al nivel "Yo simple" $(z=-3.752 ; p<.01)$ y " $E l$ otro añadido" ( $z=-3.074 ; p<.01)$.

Tabla 2. Frecuencia (FR) y duración relativa (DR) de los niveles perspectivistas para cada miembro de la tríada

\begin{tabular}{lrrrrrrrr}
\hline \multirow{2}{*}{ Niveles } & \multicolumn{2}{c}{ Madres } & \multicolumn{2}{c}{ Padres } & \multicolumn{2}{c}{ Hijos/as } & \multicolumn{2}{c}{ Total } \\
\cline { 2 - 10 } & FR & DR & FR & DR & FR & DR & \multicolumn{1}{c}{ FR } & DR \\
\hline "Yo simple" & 4.54 & 4.36 & 3.36 & 3.49 & 3.05 & 1.22 & 10.95 & 9.07 \\
\hline "Yo razonado" & 18.32 & 24.69 & 13.88 & 18.93 & 10.92 & 9.93 & 43.12 & 53.55 \\
\hline "El otro como obstáculo" & 10.96 & 10.46 & 4.13 & 4.04 & 17.49 & 12.60 & 32.58 & 27.1 \\
\hline "El otro añadido" & 5.46 & 3.51 & 4.48 & 3.02 & 0.97 & 0.40 & 10.91 & 6.93 \\
\hline "Nosotros" & 1.07 & 1.37 & 0.86 & 1.10 & 0.50 & 0.87 & 2.43 & 3.34 \\
\hline Totales & 40.36 & 44.39 & 26.71 & 30.59 & 32.93 & 25.02 & 100.00 & 100.00 \\
\hline
\end{tabular}

Aparecen diferencias significativas entre la frecuencia $(z=-2.606 ; p<.01)$ y duración $(z=-2.000 ; p<.05)$ relativa a favor de las madres respecto a los padres, y de la frecuencia $(z=-2.043 ; p<.05)$ y duración $(z=-3.146 ; p<.01)$ relativa a favor nuevamente 
de las madres respecto a los hijos. Por tanto, de forma global las madres intervienen con más frecuencia y con una mayor duración que los otros dos miembros de la tríada.

Se observan diferencias significativas en la frecuencia y la duración relativa en algunos niveles perspectivistas entre los participantes de la tríada. Las madres presentan una mayor frecuencia que los padres en el nivel "Yo razonado" ( $z=-2.027$; $p<.05)$, aportando más explicaciones a sus opiniones que los padres, pero también en el nivel “El otro como obstáculo”, utilizando más intervenciones $(z=-3.724 ; p<.01)$ y más tiempo $(z=-3.165 ; p<.01)$ para atacar los planteamientos del hijo-a que los padres.

Por otro lado, las madres destinan significativamente más tiempo a exponer, sin justificar, sus ideas que los hijos ("Yo simple", $z=-3.373 ; p<.01$ ). Aunque también utilizan el nivel "El otro añadido" más frecuentemente $(z=-4.229 ; p<.01)$ y con más duración $(z=-4.141 ; p<.01)$, empleando más intervenciones y más tiempo a tomar en cuenta el planteamiento del otro. Además, las madres aportan significativamente más explicaciones que el hijo $(z=-2.960 ; p<.01)$ y durante más tiempo $(z=-3.687 ; p<.01)$ de acuerdo con el nivel "Yo razonado”. Sin embargo, los hijos dedican más intervenciones que las madres al nivel "El otro como obstáculo" $(z=-3.985 ; p<.01)$ para oponerse a la postura del otro.

Los padres presentan una frecuencia significativamente mayor que los hijos en los dos niveles perspectivistas menos elaborados: "Yo simple" ( $z=-2.516 ; p<.01)$, y "Yo razonado" ( $z=-2.692 ; p<.01)$. No obstante los padres, al igual que las madres, emplean más intervenciones $(z=-3.300 ; p<.01)$ y más tiempo $(z=-4.012 ; p<.01)$ en el nivel "El otro añadido", siendo más capaces que los hijos de descifrar los argumentos del otro y relacionarlos con los propios. Los hijos, por su parte, destinan más intervenciones $(z=-4.682 ; p<.01)$ y más tiempo $(z=-3.946 ; p<0.01)$ que los padres al nivel "El otro como obstáculo".

Relaciones entre la frecuencia y duración de los diferentes niveles perspectivistas de los miembros de la tríada

Hemos encontrado correlaciones significativas entre los niveles perspectivistas de los miembros de la tríada, tanto para la frecuencia como para la duración relativa. A continuación explicaremos los resultados más relevantes, teniendo siempre en cuenta que las descripciones no implican una relación causal unidireccional.

Los análisis indican relación entre la frecuencia relativa de los niveles perspectivistas del padre y de la madre. Así, el nivel perspectivista "Yo simple" del padre con órdenes y oposiciones simples correlaciona negativamente con el nivel "Nosotros" de la madre $(-.397 ; p<.05)$. Asimismo cuando el nivel "Yo razonado" del padre aumenta, esto se correlaciona con una disminución del nivel básico "Yo simple" $(-.647 ; p<.01)$ y de los ataques propios del nivel "El otro como obstáculo" de la madre 
(-.612; $p<.01)$. Este último nivel también disminuye en la madre cuando el padre aumenta las intervenciones en el nivel "El otro añadido".

Por su parte, el nivel de perspectivismo más bajo "Yo simple" del hijo, se correlaciona negativamente con el "Yo razonado" de la madre $(-.448 ; p<.01)$ la cual tiende a explicar menos su propuesta, y positivamente con el nivel "El otro como obstáculo" del padre (.368; $p<.05)$, que intenta demoler los planteamientos del hijo. Además, el nivel "El otro como obstáculo" del hijo correlaciona positivamente con el "Yo simple" (.419; $p<.05)$, y "El otro como obstáculo" de la madre $(.667 ; p<.01)$; por tanto, cuanto mayor es la frecuencia de los ataques del hijo menor es el perspectivismo de la madre. En coherencia con estos resultados, cuanto menor es la frecuencia de " $E l$ otro como obstáculo" del hijo, se dan más los niveles perspectivistas del padre "Yo razonado" (-.497; $p<.01)$, “El otro añadido" (-.374; p<.05) y "Nosotros" (-.373; $p<.05)$, resultando que cuando el hijo ataca menos al padre, éste tiende a justificar más sus propuestas y a mostrar mayor perspectivismo. Por fin, respecto a los niveles perspectivistas más elaborados del hijo se observa lo siguiente: "El otro añadido" alcanza una frecuencia más elevada cuanto más busca la madre una solución conjunta aumentando el nivel "Nosotros" $(.387$; $p<.05)$; y en contrapartida, el nivel "Nosotros" del hijo disminuye cuanto más centrado está el padre en sí mismo, al aumentar su frecuencia de "Yo simple” (-.426; $p<.05)$, y "Yo razonado" $(-.440 ; p<.05)$.

En cuanto a la duración, la madre disminuye su participación en los niveles "Yo simple" (-.635; $p<.01)$, "Yo razonado" (-.525; $p<.01)$, "El otro como obstáculo" $(-.517 ; p<.01)$ y "Nosotros" $(-.400 ; p<.05)$, cuando aumenta el tiempo de las explicaciones del padre en el nivel "Yo razonado". Asimismo cuando aumenta la duración de las participaciones del hijo en el nivel "Yo simple”, la madre disminuye su participación en el nivel "Yo razonado" (-.380; $p<.05)$ ”, empleando menos tiempo para justificar sus propuestas. Este incremento del "individualismo" del hijo es combatido por el padre con un aumento de la duración de sus ataques en el nivel "El otro como obstáculo" (.440; $p<.05)$. No obstante, cuando el hijo aumenta la duración del nivel perspectivista "Yo razonado", la madre es sensible a este cambio respondiendo con una disminución del nivel "El otro como obstáculo" (-.370; $p<.01)$.

El padre, sin embargo, se sigue mostrando más beligerante siendo sensible al aumento de la duración de los ataques del hijo, respondiendo con menor tiempo en el nivel "Nosotros" (-.440; $p<.05)$. Además, cuando el padre dedica más tiempo a oponerse al hijo en el nivel el "Otro como obstáculo" (.440; $p<.05)$, la duración del nivel "El otro añadido" del hijo, considerando estratégicamente los opiniones de los otros, aumenta. Solo cuando el hijo aumenta el tiempo del nivel "Nosotros", el padre cede disminuyendo la duración los niveles "Yo simple" $(-.424 ; p<.05)$ y "Yo razonado" $(-.437 ; p<.05)$. 


\section{DISCUSIÓN Y CONCLUSIONES}

El objetivo general de este estudio ha sido conocer el nivel de perspectivismo desarrollado en situaciones de conflicto familiar entre padres e hijos adolescentes. De forma general los resultados indican un nivel discreto de perspectivismo, ya que predominan, tanto en frecuencia como en duración, los niveles "Yo razonado" y "El otro como obstáculo”. Lo cual significa que las discusiones familiares se caracterizan por la defensa de las posturas propias y los ataques a las ajenas. Siendo los adolescentes quienes más se obstinan en atacar a sus padres (Rodrigo et al., 2008), lo que se corresponde con el tópico clima relacional de confrontación durante la adolescencia inicial e intermedia de los hijos. Por tanto, es evidente que lo deseable es un aumento del perspectivismo que facilite la negociación necesaria para mejorar la convivencia familiar. Dado que los hijos en la adolescencia acaban de empezar a desarrollar estas capacidades (Correa et al., 2009), parece lógico esperar que sean los padres quienes den el primer paso perspectivista animando así a sus hijos a imitarles. De lo contrario se corre el riesgo de que ambas partes permanezcan ancladas en sus posturas (Medina et al., 2003) en ciclos reiterados de defensa y ataque (Johnson y Roloff, 2000).

En este sentido parece que tanto los padres como las madres conceden una oportunidad al perspectivismo, procurando menos ataques que los hijos, lo cual complementan las madres concentrando el discurso en más explicaciones sobre sus propuestas en comparación con los padres y los hijos. Nos referimos, sin duda, a las temidas "charlas" que los adolescentes tienen que "soportar" de sus madres. Es cierto que las madres monopolizan el discurso familiar con más intervenciones y más tiempo de intervención, y por tanto, y en general, manifiestan más registros de casi todos los niveles perspectivistas. Sin embargo, también parecen ser las mejores precursoras del perspectivismo, pudiendo interpretarse que se muestran sensibles a cualquier avance de los hijos en esta dirección. Por ejemplo, cuando los hijos amplían el tiempo de sus explicaciones, las madres podrían disminuir reactivamente la cuantía de sus ataques. Y cuando los hijos consiguen aumentar las intervenciones del nivel "El otro añadido", las madres responderían entusiastamente con más alusiones a soluciones conjuntas del nivel "Nosotros". Al mismo tiempo establecerían límites, pues cuando los hijos incrementan el egocéntrico "Yo simple", las madres reducirían el tiempo de sus explicaciones, y lo elevarían contingentemente cuando ellos acortan la duración de este nivel de nulo perspectivismo. Paralelamente reaccionarían a los ataques de los hijos con más ataques y más individualismo.

Esta sensibilidad maternal también se exhibiría con los padres pudiendo interpretarse de nuevo que restringen la frecuencia de sus contraargumentos cuando los padres dan más explicaciones o les dedican más tiempo, y cuando consideran, aunque sea de modo interesado, las posturas ajenas; al tiempo que ellas limitarían la alusión a 
soluciones conjuntas cuando los padres aumentan el nivel más primitivo "Yo Simple”. En contraste, los padres parecen mostrarse más belicosos y autoritarios, pues responderían con más ataques y de mayor duración al nivel "Yo simple" de los hijos. Y si éstos se atreven con contraargumentos los padres retrocederían disminuyendo el nivel "Nosotros". Solo cuando los hijos cejan en sus ataques, los padres consentirían en elevar su perspectivismo, incluso hasta el nivel "Nosotros". De hecho, podría pensarse que los hijos temen las reacciones de sus padres, pues basta con que éstos últimos rebajen la duración de los dos niveles más básicos "Yo simple” y "Yo razonado" para que los hijos se animen a dilatar el tiempo del nivel "Nosotros", restringiendo este nivel de avanzado perspectivismo cuando los padres, por el contrario, incrementan estas intervenciones poco perspectivistas. Quizás una forma de sortear el autoritarismo contenido en el aumento de la duración de los ataques de los padres es expresarse con mayor perspectivismo que ellos, dedicando más tiempo a razonar desde la postura de los padres de modo estratégico, para así poder persuadirles y llevarles a su terreno más fácilmente.

En definitiva, si los progenitores son quienes deben impulsar el perspectivismo en sus hijos adolescentes, los padres se reservan el papel más beligerante. Una actitud que los hijos pueden terminar por manipular a beneficio propio por medio de la persuasión, mientras que son las madres quienes más parecen promover el perspectivismo y la búsqueda de soluciones negociadas para un beneficio común, facilitando así la convivencia familiar.

\section{REFERENCIAS}

Anguera, M.T., Blanco, A. y Losada, J.L. (2001). Diseños observacionales, cuestión clave en el proceso de la metodología observacional. Metodología de las Ciencias del Comportamiento, 3(2), 135-161.

Bakeman, R. y Quera, V. (1996). Análisis de la interacción. Análisis secuencial con SDIS y GSEQ. Madrid: RA-MA.

Bakeman, R., Deckner, B. y Quera, V. (2005). Observing interaction: An introduction to sequential analysis. New York: Cambridge University Press.

Correa, N., Ceballos, E., Correa, A.D. y Batista, L. (2003). Efectos evolutivos y contextuales en la adopción de perspectivas y en la argumentación escrita. Cultura y Educación, 15(4), 343-356.

Correa, N., Rodríguez, J., Batista, L., Padrón, I. y Ceballos, E. (2009). Discurso argumentativo en episodios de conflictos entre padres e hijos adolescentes. Infancia y Aprendizaje, 32(3), 467-484.

García, M. y Peralbo, M. (2001). La adquisición de autonomía conductual durante la adolescencia: Expectativas de padres e hijos. Infancia y Aprendizaje, 24(2), 165-180.

Gottman, J.M. y Levenson, R.W. (2000). The timing of divorce: Predicting when a couple will divorce over a 14-year period. Journal of Marriage and the Family, 62, 737-745.

Hofer, M., Youniss, J. y Noack, P. (1998). Verbal interaction and development in families wtith adolescents. Greenwich, CT: Ablex. 
Hofer, M., Sassenberg, K. y Pikowsky, B. (1999). Discourse asymmetries in adolescent daughters' disputes with mothers. International Journal of Behavioral Development, 23(4), 10011022 .

Leitão, S. (2003). Evaluating and selecting counterarguments. Studies of children's rhetorical awareness. Written Communicaton, 20(3), 269-306.

Medina, F.J., Dorado, M.A., de Cisneros, I.E.J., Arévalo, A. y Munduate, L. (2003). Secuencias conductuales en la efectividad de la gestión del conflicto. Psicothema, 15(1), 12-18.

Parra, A. y Oliva, A. (2002). Comunicación y conflicto familiar durante la adolescencia. Anales de Psicología, 18(2), 215-231.

Perea, A., Alday, L. y Castellano, J. (2005). "Match Vision Studio" software para la observación deportiva. En L.M. Sautu, J. Castellano, A. Blanco, A. Hernández, A. Goñi y F. Martínez (Coords.), Evaluación e intervención en el ámbito deportivo. Diputación de Álava.

Rodrigo, M.J., García, M., Máiquez, M.L., Rodríguez, G. y Padrón, I. (2008). Estrategias y metas en la resolución de conflictos cotidianos entre adolescentes, padres y madres. Infancia y Aprendizaje, 31(3), 347-362.

Recibido: 22 de febrero de 2013

Recepción Modificaciones: 8 de marzo de 2013

Aceptado: 16 de septiembre de 2013 